\title{
Notes on three conjectures involving the digamma and generalized digamma functions
}

\section{Ladislav Matejíčka ${ }^{*}$}

\section{"Correspondence:}

ladislav.matejicka@tnuni.sk

${ }^{1}$ Faculty of Industrial Technologies in Púchov, Trenčín University of

Alexander Dubček in Trenčín,

Púchov, Slovakia

\begin{abstract}
In the paper, we solve one conjecture on an inequality involving digamma function, an open problem, and a conjecture on monotonicity of functions involving generalized digamma function. We also prove a new inequality for digamma function.

MSC: Primary 33B15; secondary 26A48; 26A51

Keywords: Digamma function; $(p, k)$-digamma function; Complete monotonicity; Monotonicity; Inequalities
\end{abstract}

\section{Introduction}

In the last years, the $(p, k)$-analogue of the gamma and polygamma functions has been studied intensively by a lot of authors. For historical background of the theory, see, for example, [1-24].

It is well known that:

- a function $f$ is said to be completely monotonic $[6,21]$ on an interval $I$ if $f$ has derivatives of all orders on $I$ and

$$
(-1)^{n} f^{(n)}(x) \geq 0
$$

for $x \in I, n \geq 0, n \in N$ (due to $0 \in N$ ).

- the Euler gamma function $[14-16,20,22,23]$ is defined by

$$
\Gamma(x)=\int_{0}^{\infty} t^{x-1} e^{-t} d t
$$

for $x>0$;

- the digamma function $[11-13,24]$ is defined by

$$
\psi(x)=\frac{\Gamma^{\prime}(x)}{\Gamma(x)}=-\gamma-\frac{1}{x}+\sum_{n=1}^{\infty} \frac{x}{n(n+x)},
$$

where $\gamma$ is the Euler-Mascheroni constant [5].

\section{Springer}


Recently, Díaz and Pariguan [4] defined the generalized gamma function

$$
\Gamma_{k}(x)=\lim _{n \rightarrow \infty} \frac{n ! k^{n}(n k)^{\frac{x}{k}-1}}{x(x+k) \cdots(x+(n-1) k)}
$$

for $k>0$ and $x \in C \backslash k Z^{-}$and the generalized digamma function

$$
\psi_{k}(x)=\frac{\Gamma_{k}^{\prime}(x)}{\Gamma_{k}(x)}=\frac{\ln (k)-\gamma}{k}-\frac{1}{x}+\sum_{n=1}^{\infty} \frac{x}{n k(n k+x)} .
$$

Very recently, Nantomah, Prempeh, and Twum [8] introduced a new definition of the $(p, k)$-gamma function

$$
\Gamma_{p k}(x)=\frac{(p+1) ! k^{p+1}(p k)^{\frac{x}{k}-1}}{x(x+k) \cdots(x+p k)}
$$

for $k>0$ and $x>0, p \geq 0, p \in N$, and the $(p, k)$-digamma function

$$
\psi_{p k}(x)=\frac{\Gamma_{p k}^{\prime}(x)}{\Gamma_{p k}(x)}=\frac{\ln (p k)}{k}-\sum_{n=0}^{p} \frac{1}{n k+x}
$$

for $k>0$ and $x>0, p \geq 0, p \in N$.

We note that

$$
\begin{array}{ll}
\lim _{k \rightarrow 1} \psi_{k}(x)=\psi(x), & \lim _{k \rightarrow 1} \Gamma_{k}(x)=\Gamma(x), \\
\lim _{p \rightarrow \infty} \Gamma_{p k}(x)=\Gamma_{k}(x), \quad \lim _{p \rightarrow \infty} \psi_{p k}(x)=\psi_{k}(x) .
\end{array}
$$

Li Yin, Li-Guo Huang, Zhi-Min Song, and Xiang Kai Dou [19] posed the following conjecture.

Conjecture 1 ([19]) For $p>0$ and $k \geq 1$, the function

$$
\phi_{p k}(x)=\psi_{p k}(x)+\ln \left(e^{\frac{1}{x}-\frac{1}{x+p k+k}}-1\right)
$$

is strictly decreasing from $(0, \infty)$ onto $\left(-\infty, \phi_{p k}(k)\right)$.

Li Yin [17] posed the following open problem.

Open Problem 1 ([17]) If the function

$$
\delta_{p k \alpha}(x)=x^{\alpha}\left[\frac{1}{k} \ln \frac{p k x}{x+k(p+1)}-\psi_{p k}(x)\right]
$$

is completely monotonic on $(0, \infty)$, then is it true that $\alpha \leq 1$ ?

Yuming Chu, Xiaoming Zhang, and Xiaoming Tang [3] posed the following conjecture. 
Conjecture 2 For $b>a>0$, we have

$$
(b-L(a, b)) \psi(b)+(L(a, b)-a) \psi(a)>(b-a) \psi(\sqrt{b a}),
$$

where $L(a, b)=(b-a) /(\ln (b)-\ln (a))$.

The goal of the paper is to solve Conjecture 1, Conjecture 2, and Open Problem 1.

\section{Methods}

In this paper, we use methods of mathematical and numerical analysis. We also use the software MATLAB for some computing.

\section{Results and discussion}

In this section, we disprove Conjecture 1 (see [19]) and Conjecture 2 (see [3]) and prove one new inequality (Theorem 1) and Open Problem 1 (see [17]).

\subsection{Disproving Conjecture 1}

It is evident that $\phi_{p k}(x)$ is strictly decreasing only if $e^{\phi_{p k}(x)}$ is strictly decreasing. We have

$$
e^{\phi_{p k}(x)}(x)=v_{p k}(x)=e^{\psi_{p k}(x+k)}-e^{\psi_{p k}(x)}
$$

Using Matlab, we obtain Table 1.

The table shows that $v_{p k}\left(x_{1}\right)<v_{p k}\left(x_{2}\right)$ for $0<x_{1}<x_{2}, p=100,000, p=100,010, k=1.1$, $k=1.6, k=2.1$. So $\phi_{p k}(x)$ is not strictly decreasing on $(0, \infty)$ for $p>0$ and $k>1$.

Remark 1 We note that Conjecture 1 (see [19]) is false since $\lim _{x \rightarrow 0^{+}} v_{p k}^{\prime}(x)>0$ for $p \geq 1$ and $k>0$.

Indeed, differentiation of $v_{p k}(x)$ yields

$$
v_{p k}^{\prime}(x)=e^{\psi_{p k}(x+k)} \psi_{p k}^{\prime}(x+k)-e^{\psi_{p k}(x)} \psi_{p k}^{\prime}(x)
$$

Because of

$$
\begin{aligned}
\lim _{x \rightarrow 0^{+}} e^{\psi_{p k}(x)} \psi_{p k}^{\prime}(x) & =\lim _{x \rightarrow 0^{+}} e^{\frac{\ln (p k)}{k}-\sum_{n=1}^{p} \frac{1}{n k+x}} e^{-\frac{1}{x}}\left(\frac{1}{x^{2}}+\sum_{n=1}^{p} \frac{1}{(n k+x)^{2}}\right) \\
& =C_{p k} \lim _{t \rightarrow+\infty} e^{-t} t^{2}=0,
\end{aligned}
$$

Table 1 Values of $p, k, x_{1}, x_{2}, v_{p k}\left(x_{1}\right), v_{p k}\left(x_{2}\right)$

\begin{tabular}{llllll}
\hline$p$ & $k$ & $x_{1}$ & $v_{p k}\left(x_{1}\right)$ & $x_{2}$ & $v_{p k}\left(x_{2}\right)$ \\
\hline 100,000 & 1.1 & 0.1 & 0.733034946365922 & 0.25 & 0.849008046429035 \\
100,000 & 1.6 & 0.1 & 0.994429244720941 & 0.25 & 1.060815738973497 \\
100,000 & 2.1 & 0.1 & 1.121247007413613 & 0.25 & 1.157039128396737 \\
100,010 & 1.1 & 0.1 & 0.733034947426004 & 0.25 & 0.849008047776431 \\
100,010 & 1.6 & 0.1 & 0.994429244720941 & 0.25 & 1.060815740083848 \\
100,010 & 2.1 & 0.1 & 1.121247008239857 & 0.25 & 1.157039129298960 \\
\hline
\end{tabular}


where $t=1 / x$, and $C_{p k}>0$ is a constant, we obtain

$$
\lim _{x \rightarrow 0^{+}} v_{p k}^{\prime}(x)=e^{\psi_{p k}(k)} \psi_{p k}^{\prime}(k)>0
$$

for $p \geq 1$ and $k>0$. This implies that, for all $k>0$ and $p \geq 1$, there is $x_{p k}>0$ such that $v_{p k}(x)$ is a strictly increasing function on $\left(0, x_{p k}\right)$. So, $\phi_{p k}(x)$ is a strictly increasing function on $\left(0, x_{p k}\right)$.

Next, by the mean value theorem we get

$$
\begin{aligned}
v_{p k}^{\prime}(x) & =e^{\psi_{p k}(x+k)} \psi_{p k}^{\prime}(x+k)-e^{\psi_{p k}(x)} \psi_{p k}^{\prime}(x) \\
& =e^{\psi_{p k}\left(\xi_{p k x}\right)}\left(\psi_{p k}^{\prime 2}\left(\xi_{p k x}\right)+\psi_{p k}^{\prime \prime}\left(\xi_{p k x}\right)\right) k=e^{\psi_{p k}\left(\xi_{p k x}\right)} w_{p k}\left(\xi_{p k x}\right) k,
\end{aligned}
$$

where $x<\xi_{p k x}<x+k$.

Due to

$$
\begin{aligned}
w_{p k}(x) & =\frac{1}{x^{4}}\left[\left(1+\sum_{n=1}^{p} \frac{x^{2}}{(n k+x)^{2}}\right)^{2}-2 x\left(1+\sum_{n=1}^{p} \frac{x^{3}}{(n k+x)^{3}}\right)\right] \\
& <\frac{1}{x^{4}}\left[(1+p)^{2}-2 x\right],
\end{aligned}
$$

we obtain that, for all $k>0$ and $p \geq 1$, the function $v_{p k}^{\prime}(x)$ is a negative function on $((1+$ $\left.p)^{2} / 2,+\infty\right)$. So, $\phi_{p k}(x)$ is a strictly decreasing function on $\left((1+p)^{2} / 2,+\infty\right)$.

Finally, computer calculations show that, for $p \geq 1$ and $k>1$, there is $0<x_{p k}<1$ such that $\phi_{p k}(x)$ is an increasing function on $\left(0, x_{p k}\right)$ and a decreasing function on $\left(x_{p k},+\infty\right)$.

\subsection{Proof of Open Problem 1}

Let $\delta_{p k \alpha}(x)$ be a completely monotonic function on $(0, \infty)$. Then $(-1)^{n} \delta_{p k \alpha}^{(n)}(x) \geq 0$ for $x \in$ $(0, \infty)$ and $\alpha \in R$. So $\delta_{p k \alpha}^{\prime}(x) \leq 0$ for $x \in(0, \infty)$. A simple computation gives

$$
\begin{aligned}
\delta_{p k \alpha}^{\prime}(x)= & \alpha x^{\alpha-1}\left[\frac{1}{k} \ln \frac{p k x}{x+k(p+1)}-\psi_{p k}(x)\right] \\
& +x^{\alpha}\left[\frac{p+1}{x(x+p k+k)}-\sum_{n=0}^{p} \frac{1}{(n k+x)^{2}}\right] \leq 0,
\end{aligned}
$$

which is equivalent to

$$
\begin{aligned}
& \alpha\left(\frac{1}{k} \ln \frac{p k x}{x+k(p+1)}-\psi_{p k}(x)\right) \\
& \quad+x\left(\frac{p+1}{x(x+p k+k)}-\sum_{n=0}^{p} \frac{1}{(n k+x)^{2}}\right) \leq 0 .
\end{aligned}
$$

Because of (see [17])

$$
\frac{1}{k} \ln \frac{p k x}{x+k(p+1)}-\psi_{p k}(x)>0
$$


we obtain

$$
\alpha \leq d(x)=\frac{x\left(-\frac{p+1}{x(x+p k+k)}+\sum_{n=0}^{p} \frac{1}{(n k+x)^{2}}\right)}{\frac{1}{k} \ln \frac{p k x}{x+k(p+1)}-\psi_{p k}(x)}
$$

for all $x>0$.

Similarly as in [1], the proof will be done if we show that

$$
\lim _{x \rightarrow 0^{+}} d(x) \leq 1
$$

Direct computation leads to

$$
\begin{aligned}
\lim _{x \rightarrow 0^{+}} d(x) & =\lim _{x \rightarrow 0^{+}} \frac{-\frac{(p+1) x}{x+p k+k}+1+\sum_{n=1}^{p} \frac{x^{2}}{(n k+x)^{2}}}{x\left[\frac{1}{k} \ln \frac{p k x}{x+k(p+1)}-\frac{\ln (p k)}{k}+\frac{1}{x}+\sum_{n=1}^{p} \frac{1}{n k+x}\right]} \\
& =\frac{1}{\lim _{x \rightarrow 0^{+}} \frac{x}{k} \ln \left(\frac{x}{x+p k+k}\right)+1+\sum_{n=1}^{p} \frac{x}{n k+x}}=1 .
\end{aligned}
$$

Indeed, $\lim _{x \rightarrow 0^{+}} d(x)=1$ implies that, for each $\varepsilon>0$, there is $x_{\varepsilon}>0$ such that $d\left(x_{\varepsilon}\right)<1+\varepsilon$, so $\alpha<1+\varepsilon$, and thus $\alpha \leq 1$. This completes the proof.

\subsection{Disproving Conjecture 2}

We show that Conjecture 2 is false. Let $0<a<b$. Put $y^{2}=a / b$. Then $0<y<1$. Conjecture 2 is equivalent to

$$
\left(1+\frac{1-y^{2}}{2 \ln (y)}\right) \psi(b)-\left(\frac{1-y^{2}}{2 \ln (y)}+y^{2}\right) \psi\left(b y^{2}\right)>\left(1-y^{2}\right) \psi(b y),
$$

which can be rewritten as

$$
F(b, y)=\left(1-y^{2}+2 \ln (y)\right)\left(\psi(b)-\psi\left(b y^{2}\right)\right)-2 \ln (y)\left(1-y^{2}\right)\left(\psi(b y)-\psi\left(b y^{2}\right)\right)<0 .
$$

Let $b$ be fixed. We prove that $\lim _{y \rightarrow 0^{+}} F(b, y)=+\infty$. This implies that Conjecture 2 does not valid. Using the well-known formula

$$
\psi(x)=-\gamma-\frac{1}{x}+\sum_{n=1}^{\infty} \frac{1}{n}-\frac{1}{n+x},
$$

we obtain

$$
\psi(b)-\psi\left(b y^{2}\right)=\frac{1}{b y^{2}}-\frac{1}{b}+\sum_{n=1}^{\infty} \frac{1}{n+b y^{2}}-\frac{1}{n+b}
$$

and

$$
\psi(b y)-\psi\left(b y^{2}\right)=\frac{1}{b y^{2}}-\frac{1}{b y}+\sum_{n=1}^{\infty} \frac{1}{n+b y^{2}}-\frac{1}{n+b y} .
$$


So

$$
\begin{gathered}
F(b, y)=\frac{1}{b y^{2}}\left(1-y^{2}+2 \ln (y)\right)\left(1-y^{2}+b y^{2} \varphi_{1}(b, y)\right) \\
-\frac{2 \ln (y)\left(1-y^{2}\right)}{b y^{2}}\left(1-y+b y^{2} \varphi_{2}(b, y)\right),
\end{gathered}
$$

where

$$
0<\varphi_{1}(b, y)<\frac{b\left(1-y^{2}\right) \pi^{2}}{6} \text { and } 0<\varphi_{2}(b, y)<\frac{b y(1-y) \pi^{2}}{6} .
$$

The function $F(b, y)$ may be rearranged as

$$
\begin{aligned}
F(b, y)= & \frac{1}{b y^{2}}\left[\left(1-y^{2}\right)\left(1-y^{2}+b y^{2} \varphi_{1}(b, y)\right)\right. \\
& \left.+2 \ln (y)\left(b y^{2} \varphi_{1}(b, y)+y-y^{3}-b\left(1-y^{2}\right) y^{2} \varphi_{2}(b, y)\right)\right] .
\end{aligned}
$$

This implies that $\lim _{y \rightarrow 0^{+}} F(b, y)=+\infty$.

\subsection{Proof of Theorem 1}

Theorem 1 Let $0<a<b<4 / 10$. Then

$$
(b-L(a, b)) \psi(b)+(L(a, b)-a) \psi(a)<(b-a) \psi(\sqrt{b a}) .
$$

Proof It is easily derived that (10) is equivalent to $F(b, y)>0$, where $y^{2}=a / b, 0<y<1$, and

$$
\begin{aligned}
F(b, y)= & \left(1-y^{2}+2 \ln (y)\right)\left(\psi(b)-\psi\left(b y^{2}\right)\right) \\
& -2 \ln (y)\left(1-y^{2}\right)\left(\psi(b y)-\psi\left(b y^{2}\right)\right) .
\end{aligned}
$$

Using (8), we obtain

$$
\begin{aligned}
\psi(b)-\psi\left(b y^{2}\right) & =\frac{1-y^{2}}{b y^{2}}+b\left(1-y^{2}\right) \sum_{n=1}^{\infty} \frac{1}{\left(n+b y^{2}\right)(n+b)} \\
& <\frac{1-y^{2}}{b y^{2}}+b\left(1-y^{2}\right) \sum_{n=1}^{\infty} \frac{1}{(n+b y)^{2}}
\end{aligned}
$$

due to $\left(n+b y^{2}\right)(n+b)>(n+b y)^{2}$. So

$$
\psi(b)-\psi\left(b y^{2}\right)<b\left(1-y^{2}\right) \psi^{\prime}(b y) .
$$

Applying (9), we get

$$
\begin{aligned}
\psi(b y)-\psi\left(b y^{2}\right) & =\frac{1-y}{b y^{2}}+b y(1-y) \sum_{n=1}^{\infty} \frac{1}{\left(n+b y^{2}\right)(n+b y)} \\
& >\frac{1-y}{b y^{2}}+b(1-y) \sum_{n=1}^{\infty} \frac{1}{(n+b y)^{2}}
\end{aligned}
$$


due to $\left(n+b y^{2}\right)<(n+b y)$. So

$$
\psi(b y)-\psi\left(b y^{2}\right)>\frac{1-y}{b y^{2}}\left(1-y+b^{2} y^{3} \psi^{\prime}(b y)\right) .
$$

It is easy to see that, for $0<y<1$,

$$
s(y)=1-y^{2}+2 \ln (y)<0,
$$

which follows from $s(1)=0$ and $s^{\prime}(y)=2\left(1-y^{2}\right) / y>0$. This implies that

$$
\begin{aligned}
F(b, y)> & G(b, y) \\
= & \frac{1}{b^{2} y^{2}}\left[\left(1-y^{2}+2 \ln (y)\right) b\left(1-y^{2}\right) \psi^{\prime}(b y)\right) \\
& \left.-2 \ln (y)(1-y)\left(1-y^{2}\right)\left(1-y+b^{2} y^{3} \psi^{\prime}(b y)\right)\right] .
\end{aligned}
$$

The inequality $G>0$ is equivalent to

$$
\left.b^{2} y^{2}\left(1-y^{2}+2 \ln (y)\right) \psi^{\prime}(b y)\right)-2 \ln (y)(1-y)\left(1-y+b^{2} y^{3} \psi^{\prime}(b y)\right)>0 .
$$

Inequality (13) may be rearranged as

$$
\left.H=\psi^{\prime}(b y) b^{2} y^{2}\left(1-y^{2}+2 \ln (y)\left(1-y+y^{2}\right)\right)\right)-2 \ln (y)(1-y)^{2}>0 .
$$

Put $s_{1}(y)=1-y^{2}+2 \ln (y)\left(1-y+y^{2}\right)$. It is easy to see that $s_{1}(y)<0$ for $0<y<1$. Indeed, $s_{1}(y)<0$ is equivalent to

$$
s_{2}(y)=\frac{y^{2}-1}{1-y+y^{2}}-2 \ln (y)>0 .
$$

Due to $s_{2}(1)=0$, it suffices to show that $s_{2}^{\prime}(y)<0$.

Differentiation leads to

$$
s_{2}^{\prime}(y)=\frac{-2+3 y-2 y^{2}+3 y^{3}-2 y^{4}}{y\left(1-y+y^{2}\right)^{2}}=\frac{-(1-y)^{2}\left(2+y+2 y^{2}\right)}{y\left(1-y+y^{2}\right)^{2}}<0 .
$$

Using the well-known formula

$$
\psi^{\prime}(x)=\frac{1}{x^{2}}+\frac{1}{(1+x)^{2}}+\sum_{n=2}^{\infty} \frac{1}{(n+x)^{2}}
$$

we obtain

$$
b^{2} y^{2} \psi^{\prime}(b y)<1+\frac{b^{2} y^{2}}{(1+b y)^{2}}+b^{2} y^{2}\left(\frac{\pi^{2}}{6}-1\right) .
$$

Theorem 1 will be proved if we show

$$
\begin{aligned}
G(b)= & \left(1+\frac{b^{2} y^{2}}{(1+b y)^{2}}+b^{2} y^{2}\left(\frac{\pi^{2}}{6}-1\right)\right)\left(1-y^{2}+2 \ln (y)\left(1-y+y^{2}\right)\right) \\
& -2 \ln (y)(1-y)^{2}>0
\end{aligned}
$$


for $0<b<4 / 10,0<y<1$. Based on

$$
\frac{d G(b)}{d b}=\left(1-y^{2}+2 \ln (y)\left(1-y+y^{2}\right)\right)\left(\frac{2 b y^{2}}{(1+b y)^{3}}+2 b y^{2}\left(\frac{\pi^{2}}{6}-1\right)\right)<0,
$$

it suffices to prove that $G(0.4)>0$.

The inequality $G(b)>0$ is equivalent to

$$
2 \ln (y) f(b, y)+g(b, y)>0,
$$

where

$$
\begin{aligned}
f(b, y)= & \left(1-y+y^{2}\right)\left[(1+b y)^{2}+b^{2} y^{2}\left(1+(1+b y)^{2}\left(\frac{\pi^{2}}{6}-1\right)\right)\right] \\
& -(1+b y)^{2}(1-y)^{2}, \\
g(b, y)= & \left(1-y^{2}\right)\left[(1+b y)^{2}+b^{2} y^{2}\left(1+(1+b y)^{2}\left(\frac{\pi^{2}}{6}-1\right)\right)\right] .
\end{aligned}
$$

It is clearly seen that $f(b, y)>0$. So (14) will be done if we prove

$$
h(b, y)=2 \ln (y)+\frac{g(b, y)}{f(b, y)}>0
$$

for $b=4 / 10$ and $0<y<1$. Because of $h(0.4,1)=0$, it suffices to show that $(d h / d y)(0.4, y)<0$ for $0<y<1$. We get

$$
\frac{d h(0.4, y)}{d y}=\frac{2}{y}+\frac{\frac{d g(0.4, y)}{d y} f(0.4, y)-g(0.4, y) \frac{d f(0.4, y)}{d y}}{f^{2}(0.4, y)}<0 .
$$

Inequality (15) is equivalent to

$$
u(y)=2 f^{2}(0.4, y)+y\left(\frac{d g(0.4, y)}{d y} f(0.4, y)-g(0.4, y) \frac{d f(0.4, y)}{d y}\right)<0 .
$$

Put $a(y)=100 u(y)$. Using Taylor's series and Matlab, we obtain

$$
\begin{aligned}
a(y)= & (y-1)^{2}(899.80856587904507327359937090692(y-1) \\
& -34.541951843593497556069703611525 \\
& +2803.0064998600206956003380918157(y-1)^{2} \\
& +3449.9649390326664508411882274417(y-1)^{3} \\
& +2382.8365919732490773391558060343(y-1)^{4} \\
& +1077.4495988779774279297213875464(y-1)^{5} \\
& +341.35184858325869449609722858626(y-1)^{6} \\
& +75.928581022558019892100963581518(y-1)^{7} \\
& +11.561798822762785643694391575539(y-1)^{8}
\end{aligned}
$$




$$
\begin{aligned}
& +1.1176206646533878730984733813133(y-1)^{9} \\
& \left.\left.+0.05451808120260428649260845762504(y-1)^{10}\right)\right) \\
= & (y-1)^{2} k(y),
\end{aligned}
$$

where

$$
\begin{aligned}
k(y)= & 899.80856587904507327359937090692(y-1) \\
& -34.541951843593497556069703611525 \\
& +2803.0064998600206956003380918157(y-1)^{2} \\
& +3449.9649390326664508411882274417(y-1)^{3} \\
& +2382.8365919732490773391558060343(y-1)^{4} \\
& +1077.4495988779774279297213875464(y-1)^{5} \\
& +341.35184858325869449609722858626(y-1)^{6} \\
& +75.928581022558019892100963581518(y-1)^{7} \\
& +11.561798822762785643694391575539(y-1)^{8} \\
& +1.1176206646533878730984733813133(y-1)^{9} \\
& +0.05451808120260428649260845762504(y-1)^{10} .
\end{aligned}
$$

It is easy to see that $k(y)<k k(y)$, where

$$
\begin{aligned}
k k(y)= & 899.80856587904507327359937090692(y-1) \\
& -34.541951843593497556069703611525 \\
& +2803.0064998600206956003380918157(y-1)^{2} \\
& +3449.9649390326664508411882274417(y-1)^{3} \\
& +2382.8365919732490773391558060343(y-1)^{4} \\
& +1077.4495988779774279297213875464(y-1)^{5} \\
& +341.35184858325869449609722858626(y-1)^{6} \\
& +75.928581022558019892100963581518(y-1)^{7} \\
& +11.561798822762785643694391575539(y-1)^{8} \\
& +1.1176206646533878730984733813133(y-1)^{9} \\
& +0.05451808120260428649260845762504(y-1)^{6} .
\end{aligned}
$$

To prove that $k k(y)<0$, it suffices to show that $k k(0) \leq 0, k k(1) \leq 0, k k^{\prime}(0) \leq 0, k k^{\prime \prime}(1)>0$, $k k^{\prime \prime}(0)<0$, and $k k^{\prime \prime}(y)$ is an increasing function on $(0,1)$.

Put $c(y)=k k^{\prime \prime}(y)$. Direct computation yields

$$
\begin{aligned}
c(y)= & (22,759,659,395,315,613 y) / 1,099,511,627,776 \\
& +\left(2,021,889,693,856,018,983(y-1)^{2}\right) / 70,368,744,177,664
\end{aligned}
$$




$$
\begin{aligned}
& +\left(23,693,367,246,178,465(y-1)^{3}\right) / 1,099,511,627,776 \\
& +\left(738,027,641,132,151,741,645(y-1)^{4}\right) / 72,057,594,037,927,936 \\
& +\left(112,202,976,768,737,799(y-1)^{5}\right) / 35,184,372,088,832 \\
& +\left(11,324,961,019,967,769(y-1)^{7}\right) / 140,737,488,355,328 \\
& -8,297,891,458,330,007 / 549,755,813,888
\end{aligned}
$$

We now show that $c c(y)=k k^{\prime \prime \prime}(y)>0$. We have

$$
\begin{aligned}
c c(y)= & 57,465.561379104286260144363041036 y \\
& +64,646.975932678647041029762476683(y-1)^{2} \\
& +40,968.763999735354855158409037585(y-1)^{3} \\
& +15,945.002014737184339310260838829(y-1)^{4} \\
& +563.28081498530752213582672993653(y-1)^{6} \\
& -36,765.771744908288582109889830463 .
\end{aligned}
$$

Differentiation yields

$$
\begin{aligned}
c c^{\prime \prime}(y)= & 16,898.424449559224740369245409966 y^{4} \\
& -67,593.697798236898961476981639862 y^{3} \\
& +292,730.57087420154857682064175606 y^{2} \\
& -204,461.16215351717255543917417526 y \\
& +91,719.816493350586824817582964897
\end{aligned}
$$

Using the Cardano formula and Matlab, we get that there are no real roots of $c c^{\prime \prime}(y)=0$. Due to $c c(0)>0$, we obtain $c c^{\prime \prime}(y)>0$.

We now show that $v(y)>0$ for $0<y<1$, where $v(y)$ is a tangent line to the function $c c(y)$ at the point $(0.22, c c(0.22))$.

Using Matlab, we have

$$
c c(0.22)=1794.965061908937 \quad \text { and } \quad c c^{\prime}(0.22)=149.7626334452943 .
$$

This implies that

$$
v(y)=1794.965061908937+149.7626334452943(y-0.22) .
$$

Direct computation yields:

$$
\begin{aligned}
& v(0)=1762.017282550972, \quad v(1)=1911.779915996267, \\
& k k(0)=-2.3294 \mathrm{e}-14, \quad k k(1)=-34.5420, \quad k k^{\prime}(0)=-53.5347, \\
& k k^{\prime \prime}(0)=-937.2660, \quad k k^{\prime \prime}(1)=5.6060 \mathrm{e}+03 .
\end{aligned}
$$

This completes the proof. 


\subsection{Open problem}

Finally, we give an open problem.

Open Problem 2 Find the best possible real positive constants $b_{0}, b_{1}$ such that if $0<a<$ $b \leq b_{0}$, then

$$
(b-L(a, b)) \psi(b)+(L(a, b)-a) \psi(a)<(b-a) \psi(\sqrt{b a})
$$

and if $0<b_{1} \leq a<b$, then

$$
(b-L(a, b)) \psi(b)+(L(a, b)-a) \psi(a)>(b-a) \psi(\sqrt{b a}),
$$

where $L(a, b)=(b-a) /(\ln (b)-\ln (a))$.

Note 1 Note that our work and [3] show that $4 / 10 \leq b_{0}$ and $2 \geq b_{1}$.

\section{Conclusion}

In this paper, we proved e Open Problem 1 [17] and disproved Conjectures 1 and 2 [3, 19]. We also proved a new inequality (Theorem 1) for the digamma function. Finally, we proposed an Open Problem 2.

\section{Acknowledgements \\ The author would like to thank the editor and the anonymous referees for their valuable suggestions and comments, which helped me to improve this paper greatly. The author thanks Professor Ondrušová, FPT TnUAD, Slovakia, for his kind grant support. \\ Funding \\ The work was supported by VEGA grant No. 1/0649/17, VEGA grant No. 1/0185/19, VEGA grant No. 1/0589/17 and by Kega grant No. 007 TnUAD-4/2017.}

\section{Competing interests}

The author declares that he has no competing interests.

\section{Authors' contributions}

The author completed the paper and approved the final manuscripts.

\section{Publisher's Note}

Springer Nature remains neutral with regard to jurisdictional claims in published maps and institutional affiliations.

Received: 24 October 2018 Accepted: 4 December 2018 Published online: 12 December 2018

\section{References}

1. Alzer, H.: On some inequalities for the gamma and psi function. Math. Comput. 66, 373-389 (1997)

2. Alzer, H.: Sharp inequalities for the harmonic numbers. Expo. Math. 24(4), 385-388 (2006)

3. Chu, Y., Zhang, X., Tang, X.: An elementary inequality for psi function. Bull. Inst. Math. Acad. Sin. (N.S.) 3(3), 373-380 (2008)

4. Díaz, R., Pariguan, E.: On hypergeometric functions and Pochhammer k-symbol. Divulg. Mat. 15(2), 179-192 (2007)

5. Huang, T.-R., Han, B.-W., Ma, X.-Y., Chu, Y.-M.: Optimal bounds for the generalized Euler-Mascheroni constant. J. Inequal. Appl. 2018, Article ID 118 (2018)

6. LV, Y.-P., Sun, T.-C., Chu, Y.-M.: Necessary and sufficient conditions for a class of functions and their reciprocals to be logarithmically completely monotonic. J. Inequal. Appl. 2011, Article ID 36 (2011)

7. Nantomah, K., Merovci, F., Nasiru, S.: Some monotonic properties and inequalities for the $(p, q)$-gamma function. Kragujev. J. Math. 42(2), 287-297 (2018)

8. Nantomah, K., Prempeh, E., Twum, S.B.: On a $(p, k)$-analogue of the gamma function and some associated inequalities. Moroccan J. Pure Appl. Anal. 2(2), 79-90 (2016)

9. Qi, F., Guo, B.-N.: An alternative proof of Elezović-Giordano-Pečarić's theorem. Math. Inequal. Appl. 14(1), 73-78 (2011)

10. Qi, F., Guo, B.-N., Chen, C.-P.: Some completely monotonic functions involving the gamma and polygamma functions. J. Aust. Math. Soc. 80, $81-88$ (2006) 
11. Wang, M.-K., Chu, Y.-M.: Landen inequalities for a class of hypergeometric functions with applications. Math. Inequal. Appl. 21(2), 521-537 (2018)

12. Wang, M.-K., Li, Y.-M., Chu, Y.-M.: Inequalities and infinite product formula for Ramanujan generalized modular equation function. Ramanujan J. 46(1), 189-200 (2018)

13. Yang, Z.-H., Chu, Y.-M., Zhang, X.-H.: Sharp bounds for psi function. Appl. Math. Comput. 268, 1055-1063 (2015)

14. Yang, Z.-H., Qian, W.-M., Chu, Y.-M., Zhang, W.: On rational bounds for the gamma function. J. Inequal. Appl. 2017 Article ID 210 (2017)

15. Yang, Z.-H., Qian, W.-M., Chu, Y.-M., Zhang, W.: On approximating the error function. Math. Inequal. Appl. 21(2), 469-479 (2018)

16. Yang, Z.-H., Zhang, W., Chu, Y.-M.: Sharp Gautschi inequality for parameter $0<p<1$ with applications. Math. Inequal. Appl. 21(4), 1107-1120 (2017)

17. Yin, L.: Complete monotonicity of a function involving the $(p, k)$-digamma function. Int. J. Open Probl. Comput. Sci. Math. 11(2), 103-108 (2018)

18. Yin, L., Huang, L.-G., Lin, X.-L., Wang, Y.-L.: Monotonicity, concavity, and inequalities related to the generalized digamma function. Adv. Differ. Equ. 2018, Article ID 246 (2018)

19. Yin, L., Huang, L.-G., Song, Z.-M., Dou, X.K.: Some monotonicity properties and inequalities for the generalized digamma and polygamma functions. J. Inequal. Appl. 2018, Article ID 249 (2018). https://doi.org/10.1186/s13660-018-1844-2

20. Zhang, X.-M., Chu, Y.-M.: A double inequality for gamma function. J. Inequal. Appl. 2009, Article ID 503782 (2009)

21. Zhao, T.-H., Chu, Y.-M.: A class of logarithmically completely monotonic functions associated with a gamma function. J. Inequal. Appl. 2010, Article ID 392431 (2010)

22. Zhao, T.-H., Chu, Y.-M., Jiang, Y.-P.: Monotonic and logarithmically convex properties of a function involving gamma functions. J. Inequal. Appl. 2009, Article ID 728612 (2009)

23. Zhao, T.-H., Chu, Y.-M., Wang, H.: Logarithmically complete monotonicity properties relating to the gamma function. Abstr. Appl. Anal. 2011, Article ID 896483 (2011)

24. Zhao, T.-H., Wang, M.-K., Chu, Y.-M.: Quadratic transformation inequalities for Gaussian hypergeometric function. J. Inequal. Appl. 2018, Article ID 251 (2018)

\section{Submit your manuscript to a SpringerOpen ${ }^{\circ}$ journal and benefit from:}

- Convenient online submission

- Rigorous peer review

- Open access: articles freely available online

- High visibility within the field

- Retaining the copyright to your article

Submit your next manuscript at $\gg$ springeropen.com 